\title{
Point mutation in mitochondrial tRNA gene is associated with polycystic ovary syndrome and insulin resistance
}

\author{
YU DING $^{1}$, GUANGCHAO ZHUO ${ }^{1}$ CAIJUAN ZHANG ${ }^{1,2}$ and JIANHANG LENG ${ }^{1}$ \\ ${ }^{1}$ Central Laboratory and ${ }^{2}$ Department of Obstetrics and Gynecology, \\ Hangzhou First People's Hospital Affiliated to Nanjing Medical University, Hangzhou, Zhejiang 310006, P.R. China
}

Received March 25, 2015; Accepted January 20, 2016

DOI: $10.3892 / \mathrm{mmr} .2016 .4916$

\begin{abstract}
Polycystic ovarian syndrome (PCOS) is characterized by chronic anovulation, hyperandrogenism and polycystic ovaries. To date, the molecular mechanisms underlying PCOS have remained to be fully elucidated. As recent studies have revealed a positive association between mitochondrial dysfunction and PCOS, current investigations focus on mutations in the mitochondrial genome of patients with POCS. The present study reported a Chinese patient with PCOS. Sequence analysis of the mitochondrial genome showed the presence of homoplasmic ND5 T12338C and tRNA ${ }^{\text {Ser (UCN) }}$ C7492T mutations as well as a set of polymorphisms belonging to the human mitochondrial haplogroup F2. The T12338C mutation is known to decrease the ND5 mRNA levels and to inhibit the processing of RNA precursors. The C7492T mutation, which occurred at the highly conserved nucleotide in the anticodon stem of the tRNA ${ }^{\mathrm{Ser}(\mathrm{UCN})}$ gene, is important for the tRNA steady-state level as well as the aminoacylation ability. Therefore, the combination of the ND5 T12338C and tRNA $^{\text {Ser (UCN) }}$ C7492T mutations may lead to mitochondrial dysfunction, and is likely to be involved in the pathogenesis of PCOS. The present study provided novel insight into the molecular mechanisms of PCOS.
\end{abstract}

\section{Introduction}

Polycystic ovary syndrome (PCOS) is the most common endocrinopathy in reproductive-age women, affecting 8-18\% (1). Insulin resistance (IR) is considered to be the main pathological factor responsible for the hormonal disturbances associated with the syndrome (2), and IR in PCOS patients confers a

Correspondence to: Dr Yu Ding or Dr Jianhang Leng, Central Laboratory, Hangzhou First People's Hospital Affiliated to Nanjing Medical University, 261 Huan Sha Road, Hangzhou, Zhejiang 310006, P.R. China

E-mail: dingyu.zj@gmail.com

E-mail: lengjh5@163.com

Key words: polycystic ovary syndrome, mitochondrial DNA mutation, dysfunction, ND5, tRNA ${ }^{\mathrm{Ser}(\mathrm{UCN})}$ substantial risk for developing type 2 diabetes and cardiovascular diseases $(3,4)$. Furthermore, IR has been reported to be linked with reduced mitochondrial respiration (5). Reduced expression of nuclear-encoded genes involved in oxidative phosphorylation (OXPHOS) has been reported in skeletal muscle of patients with PCOS (6). In addition, analysis of oxygen consumption in blood mononuclear cells (leukocytes) indicated that in women with PCOS, mitochondrial complex I respiration is reduced compared with that in age- and body mass index-matched control subjects (7). Due to this central role of mitochondrial impairment in PCOS, current studies focus on mutations in the mitochondrial genome of patients with POCS.

With the purpose of elucidating the molecular basis of PCOS, the present study performed a systematic and extensive mutational screening for pathogenic mutations in the mitochondrial genome of a patient with PCOS. Previous studies by our group showed that mitochondrial OXPHOS complexes are hot spots for mutations associated with PCOS, as well as several mitochondrial transfer (mt-t)RNA mutations $(8,9)$. These tRNA mutations included tRNA ${ }^{\mathrm{Gln}} \mathrm{T} 4395 \mathrm{C}$, tRNA $^{\text {Cys }}$ G5821 A, tRNA ${ }^{\text {Asp }}$ A7543G, tRNA ${ }^{\text {Lys }}$ A8343G, tRNA $^{\text {Arg }}$ T10454C and tRNA ${ }^{\text {Glu }}$ A14693G. These mt-tRNA mutations were localized at highly conserved nucleotides, which caused structural and functional alternations, and consequently resulted a failure of mt-tRNA metabolism. The present study reported on the clinical and molecular characterization of a Han Chinese patient with PCOS-IR. A sequence analysis of the mitochondrial genome showed the

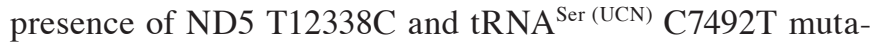
tions.

\section{Subject and methods}

Case subject. A female patient (age, 31 years) from the Hangzhou area of Zhejiang Province (China) was referred to the Department of Obstetrics and Gynecology (Hangzhou First People's Hospital, Hangzhou, China) due to an irregular menstrual cycle and suspicion of PCOS. Following obtainment of written informed consent from the patient, blood samples were obtained and a clinical evaluation was performed following protocols approved by the Ethics Committee of Hangzhou First People's Hospital (Hangzhou, China). The patient underwent a thorough physical examination, a 
laboratory assessment of metabolic syndrome and routine electrocardiography. In addition, 200 age-matched control subjects (average age, 30 years) were selected from a panel of unaffected women of Han Chinese ancestry from the same region. A thorough analysis of the patient's personal and the patient's family's medical history was performed, the patient's symptoms were assessed and PCOS was diagnosed according to the Rotterdam criteria (10): i) Clinical signs of hyperandrogenism; ii) polycystic ovaries symptom; iii) A ratio of luteinizing hormone $(\mathrm{LH})$ levels vs. follicle stimulating hormone $(\mathrm{FSH})$ of $>2.0$, or total testosterone levels of $>2.64 \mathrm{nmol} / \mathrm{l}$. In addition, a comprehensive physical examination of the patient was performed to confirm the absence of any other syndrome, including hyperprolactinemia, thyroid and adrenal diseases, 21-hydroxylase deficiency and androgen-secreting tumors. The patient had no family history of PCOS.

Laboratory assessment. Serum levels of luteinizing hormone (LH), follicle-stimulating hormone (FSH), estradiol, progesterone, total testosterone (TT) and fasting insulin levels were measured by electrochemiluminescence immunoassays (Roche Diagnostics, Basel, Switzerland). The plasma glucose levels were measured using a Beckman glucose analyzer (Ramcon, Fullerton, CA, USA). Furthermore, an oral glucose tolerance test was performed. In brief, a blood sample was obtained from the antecubital vein at 0 and $120 \mathrm{~min}$ for measurement of plasma glucose concentrations, with 0 min denoting the level of fasting plasma glucose. Patients with a plasma glucose concentration of $<7.8 \mathrm{mmol} / 1$ at the 120 -min time point were categorized as having normal glucose tolerance, while those with plasma glucose levels of 7.8-11.1 mmol/1 were classified as having impaired glucose tolerance, and those with glucose levels of $>11.1 \mathrm{mmol} / \mathrm{l}$ were indicated to have diabetes mellitus. The main outcome measure of IR was calculated using the homeostatic model assessment of IR (HOMA-IR), where a value of $>3.16$ denotes IR according to the following formula:

$$
\text { HOMA-IR }=\frac{\text { Fasting glucose } X \text { Insulin }}{22.5}
$$

Molecular and genetic analysis. Total DNA was isolated from peripheral blood leukocytes of the patient using an Universal Genomic DNA Extraction kit version 3.0 (Takara Bio Inc., Otsu, Japan). The entire mtDNA was amplified in 24 overlapping fragments as previously described (11), using 24 overlapping primers provided by the BGI (Shenzhen, China). The PCR mixture included $200 \mu \mathrm{M}$ dNTPs, $2 \mu \mathrm{l}$ buffer (10X), $0.2 \mu \mathrm{l}$ Taq DNA polymerase and $15 \mathrm{mmol} / 1 \mathrm{Mg} 2^{+}$ (Takara Bio Inc., Otsu, Japan). After polymerase chain reaction amplification, the fragments were purified and subsequently analyzed by direct sequencing in an ABI 3730 automatic DNA sequencer (Thermo Fisher Scientific, Inc., Waltham, MA, USA) using the BigDye Terminator Cycle Sequencing kit (Sigma-Aldrich, St. Louis, MO, USA). Sample sequences were compared with the revised Cambridge Reference Sequence (GenBank accession no. NC_012920) from MITOMAP, a human mitochondrial genome database (http://www.mitomap.org/MITOMAP) (12).
Table I. Clinical and biochemical parameters of the patient with polycystic ovary syndrome.

\begin{tabular}{lrc}
\hline Parameter & Value & Normal range \\
\hline LH $(\mathrm{IU} / \mathrm{l})$ & 14.54 & $1.50-9.30$ \\
FSH $(\mathrm{IU} / \mathrm{l})$ & 5.17 & $1.40-18.00$ \\
Estradiol $(\mathrm{pmol} / \mathrm{l})$ & 205.60 & $0.00-198.20$ \\
Progesterone $(\mathrm{nmol} / \mathrm{l})$ & 3.12 & $0.89-3.88$ \\
Total testosterone $(\mathrm{nmol} / \mathrm{l})$ & 2.89 & $8.36-28.70$ \\
Prolactin $(\mu \mathrm{g} / \mathrm{l})$ & 5.22 & $2.00-17.00$ \\
OGTT $(0 \mathrm{~h})(\mathrm{mmol} / \mathrm{l})$ & 5.11 & $3.90-6.10$ \\
OGTT $(2 \mathrm{~h})(\mathrm{mmol} / \mathrm{l})$ & 8.55 & - \\
Insulin $(0 \mathrm{~h})(\mu \mathrm{U} / \mathrm{ml})$ & 9.80 & $2.60-12.00$ \\
HOMA-IR $(\mathrm{x})$ & 3.72 & - \\
\hline
\end{tabular}

LH, luteinizing hormone; FSH, follicle-stimulating hormone; OGTT, oral glucose tolerance test; HOMA-IR, homeostatic model assessment of insulin resistance.

\section{Results and Discussion}

Laboratory examination of the patient showed that the levels of TT were $2.89 \mathrm{nmol} / \mathrm{l}$, and the LH/FSH ratio was 2.81. In addition, the patient was insulin resistant. The clinical laboratory parameters of the PCOS patient are listed in Table I.

Mutational screening of the mitochondrial genome showed the presence of the homoplasmic ND5 T12338C and

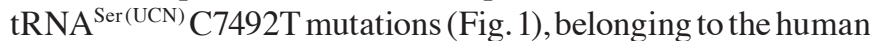
mitochondrial haplogroup F2 (13). Notably, the homoplasmic ND5 T12338C mutation was only detected in the patient with PCOS but not in the healthy controls. This mutation is a result of a replacement of the first amino acid, a translation-initiating methionine, with a threonine in the ND5 polypeptide (Fig. 2). The encoding sequence of the first methionine in the ND5 gene is extraordinarily conserved among species and organelles ranging from bacteria to human mitochondria (14). As a result, the truncated ND5 protein was expected to be shortened by two amino acids, since the ND5 T12338C mutation replaced the first amino acid from methionine to threonine and, as methionine is the translational initiation code, the mutant ND5 translates from the third methionine. As reported by a previous study, the ND5 T12338C mutation was also located in two nucleotides adjacent to the $3^{\prime}$ end of the tRNA $^{\text {Leu (CUN) }}$ gene (15). Consequently, this mutation altered respiratory function, as well as the processing of RNA precursors, thereby leading to a reduction in tRNA ${ }^{\text {Leu (CUN) }}$ levels. The functional significance of the ND5 T12338C mutation in terms of mitochondrial physiology was further supported by studies reporting that the $\mathrm{T} 12338 \mathrm{C}$ mutation may modulate the phenotypic expression of the deafness-associated 12S rRNA A1555G mutation, and that it was associated with essential hypertension and Leber's hereditary optic neuropathy $(16,17)$. The homoplasmic ND1 T3308C mutation has been suggested to contribute to the higher penetrance of hearing loss in a large African pedigree compared with that in Japanese and French pedigrees carrying the tRNA ${ }^{\text {Ser }(U C N)}$ T7511C mutation $(18,19)$. A significant reduction in steady-state levels of ND1 mRNA 


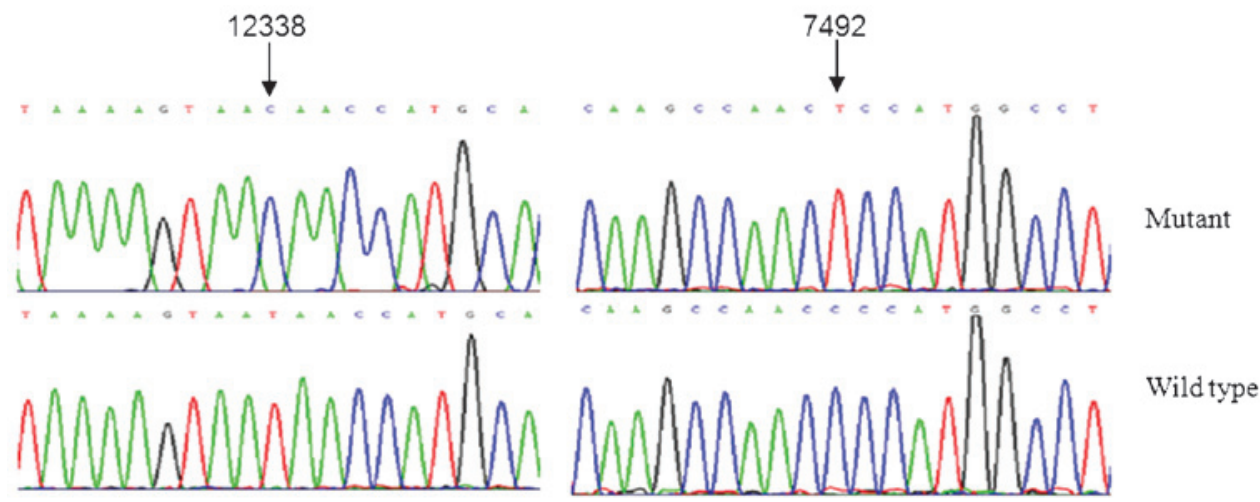

Figure 1. Identification of the T12338C and C7492T mutations in the mitochondrial genome. Partial sequence of the ND5 and tRNA ${ }^{\mathrm{Ser}(\mathrm{UCN})}$ genes from the patient and controls. Arrows indicate the positions of 12,338 and 7,492 bp.

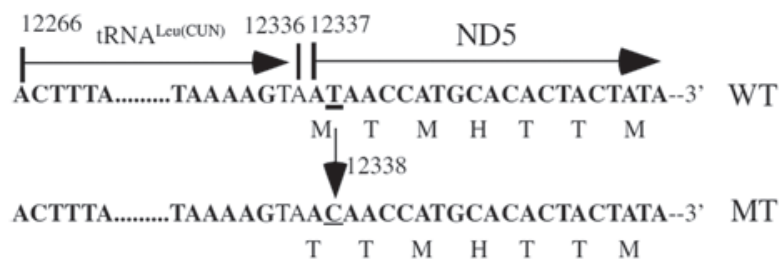

Figure 2. Scheme of the mitochondrial DNA sequence at $12,338 \mathrm{bp}$ and the adjacent sequence of ND5 and tRNA ${ }^{\text {Leu (CUN) }}$ in the wild and mutant types. Arrows indicate the position of the T12338C mutation. WT, wild-type; MT, mutant type.

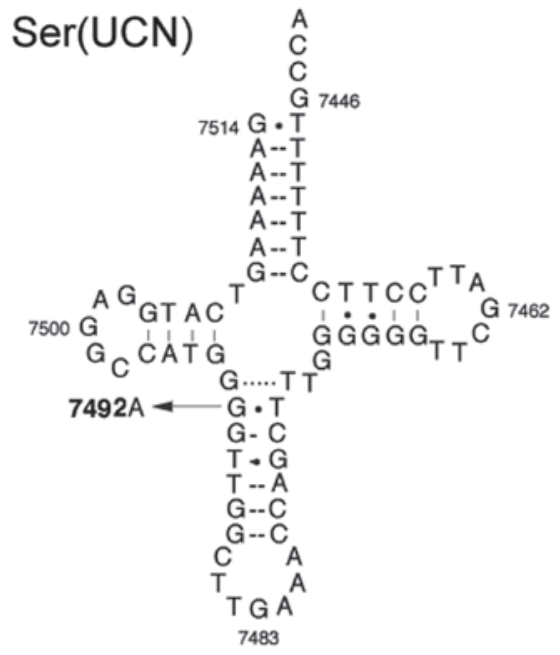

Figure 3. Secondary structure of the gene encoding mitochondrial tRNA ${ }^{\mathrm{Ser}(\mathrm{UCN})}$. The arrow indicates the C7492T mutation.

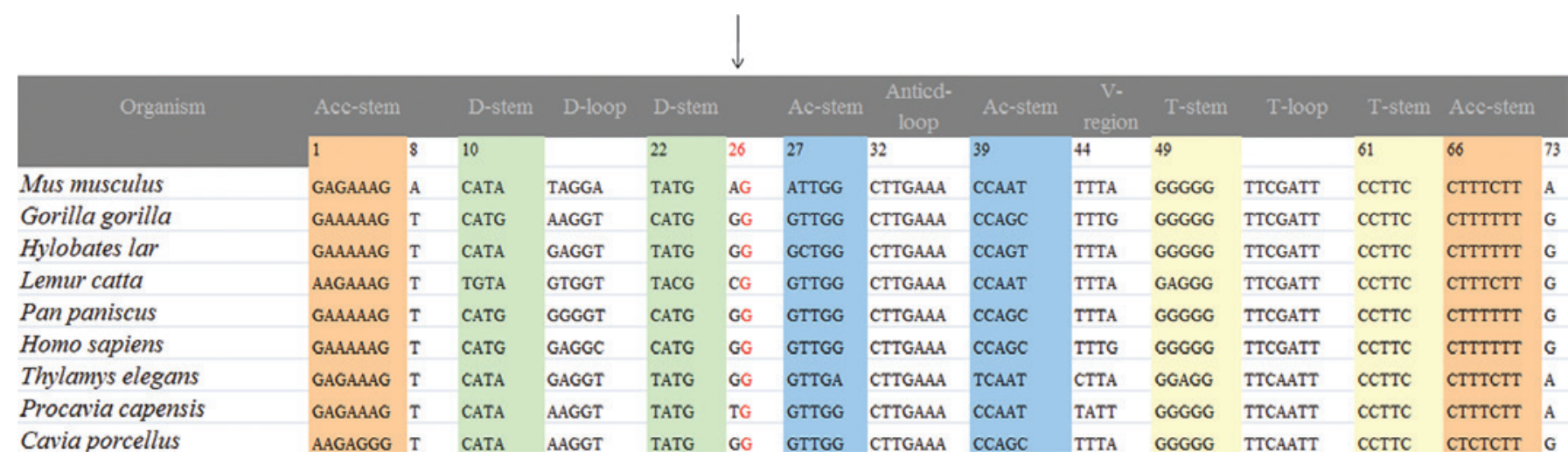

Figure 4. Alignment of tRNA ${ }^{\text {Ser (UCN) }}$ gene in various species. The arrow indicates position 26, corresponding to the C7492T mutation. 
and the adjacent tRNA ${ }^{\text {Leu (UUR) }}$ observed in cybrids carrying the $\mathrm{T} 3308 \mathrm{C}$ mutation was likely due to an alteration in the processing of the $\mathrm{H}$-strand polycistronic RNA precursors or the destabilization of ND1 mRNA (20). Thus, the ND5 T12338C mutation, which is similar to the ND1 T3308C mutation (14,19), may induce a reduction in ND5 mRNA expression levels, as well as the steady state level of tRNA ${ }^{\text {Leu (CUN); }}$ therefore, this mutation may cause mitochondrial dysfunction and, since mitochondrial dysfunction is associated with the pathogenesis of PCOS-IR (6,7), we propose the ND5 T12338C mutation is associated with PCOS-IR.

The homoplasmic C7492T mutation occurred at position 26 in the anticodon stem of the tRNA ${ }^{\text {Ser (UCN) }}$-encoding gene (Fig. 3). Mutation at this position is highly conserved among various species, which suggests that it may be associated with the pathogenesis of PCOS-IR (Fig. 4). In addition, the $\mathrm{C} 7492 \mathrm{~T}$ mutation led to an alteration of base pairing (A26-U44), and identical base pairing at the same position in tRNA ${ }^{\text {Ile }}$ generated by the heteroplasmic T4295C mutation has been associated with altered tRNA metabolism in chronic progressive external ophthalmoplegia (21). Therefore, it can be anticipated that the $\mathrm{C} 7492 \mathrm{~T}$ mutation may alter the tertiary structure of tRNA ${ }^{\mathrm{Ser}(\mathrm{UCN})}$ and, since the anticodon stem is critical for codon and anticodon interaction, this mutation may reduce the steady state level of tRNA ${ }^{\mathrm{Ser}(\mathrm{UCN})}$ as well as the aminoacylation ability. The resultant shortage of tRNA ${ }^{\text {Ser (UCN) }}$ may be responsible for defects in mitochondrial protein synthesis. Consequently, the deficiency in respiratory chain function will cause a reduction in ATP synthesis and increase the generation of reactive oxygen species. Therefore, this mutation may have an active role in the pathogenesis of PCOS-IR. However, due to the complex molecular mechanisms of PCOS, it is likely that the C7492T and $\mathrm{T} 12338 \mathrm{C}$ mutations alone are insufficient to produce the clinical phenotype; other factors, including nuclear genes, epigenetic modifications and environmental factors may contribute to the pathogenesis of PCOS.

\section{Acknowledgements}

The authors would like to thank the patient for participating this study. This work was supported by grants from the Ministry of Public Health of Zhejiang Province (grant no. 2013KYA158) and the Hangzhou Bureau of Science and Technology (grant no. 20150633B16).

\section{References}

1. March WA, Moore VM, Willson KJ, Phillips DI, Norman RJ and Davies MJ: The prevalence of polycystic ovary syndrome in a community sample assessed under contrasting diagnostic criteria. Hum Reprod 25: 544-551, 2010.

2. Dunaif A: Insulin resistance and the polycystic ovary syndrome: Mechanism and implications for pathogenesis. Endocr Rev 18: 774-800, 1997.

3. Teede HJ, Hutchison SK and Zoungas S: The management of insulin resistance in polycystic ovary syndrome. Trends Endocrinol Metab 18: 273-279, 2007.
4. Moran LJ, Misso ML, Wild RA and Norman RJ: Impaired glucose tolerance, type 2 diabetes and metabolic syndrome in polycystic ovary syndrome: A systematic review and metaanalysis. Hum Reprod Update 16: 347-363, 2010.

5. Petersen KF and Shulman GI: Etiology of insulin resistance. Am J Med 119 (5 Suppl 1): S10-S16, 2006.

6. Skov V, Glintborg D, Knudsen S, Jensen T, Kruse TA, Tan Q, Brusgaard K, Beck-Nielsen H and Højlund K: Reduced expression of nuclear-encoded genes involved in mitochondrial oxidative metabolism in skeletal muscle of insulin-resistant women with polycystic ovary syndrome. Diabetes 56: 2349-2355, 2007.

7. Victor VM, Rocha M, Bañuls C, Sanchez-Serrano M, Sola E, Gomez M and Hernandez-Mijares A: Mitochondrial complex I impairment in leukocytes from polycystic ovary syndrome patients with insulin resistance. J Clin Endocrinol Metab 94: 3505-3512, 2009.

8. Zhuo G, Ding Y, Feng G, Yu L and Jiang Y: Analysis of mitochondrial DNA sequence variants in patients with polycystic ovary syndrome. Arch Gynecol Obstet 286: 653-659, 2012.

9. Zhuo G, Feng G, Leng J, Yu L and Jiang Y: A 9-bp deletion homoplasmy in women with polycystic ovary syndrome revealed by mitochondrial genome-mutation screen. Biochem Genet 48: 157-163, 2010.

10. Rotterdam ESHRE/ASRM-Sponsored PCOS Consensus Work-shop Group: Revised 2003 consensus on diagnostic criteria and long-term health risks related to polycystic ovary syndrome. Fertil Steril 81: 19-25, 2004.

11. Rieder MJ, Taylor SL, Tobe VO and Nickerson DA: Automating the identification of DNA variations using quality-based fluorescence re-sequencing: Analysis of the human mitochondrial genome. Nucleic Acids Res 26: 967-973, 1998.

12. Brandon MC, Lott MT, Nguyen KC, Spolim S, Navathe SB, Baldi P and Wallace DC: MITOMAP: A human mitochondrial genome database-2004 update. Nucleic Acids Res 33 (Database issue): D611-D613, 2005.

13. Kong QP, Bandelt HJ, Sun C, Yao YG, Salas A, Achilli A, Wang CY, Zhong L, Zhu CL, Wu SF, et al: Updating the East Asian mtDNA phylogeny: A prerequisite for the identification of pathogenic mutations. Hum Mol Genet 15: 2076-2086, 2006.

14. Chen B, Sun D, Yang L, Zhang C, Yang A, Zhu Y, Zhao J, Chen Y, Guan M, Wang X, et al: Mitochondrial ND5 T12338C, tRNA(Cys) T5802C and tRNA(Thr) G15927A variants may have a modifying role in the phenotypic manifestation of deafness-associated $12 \mathrm{~S}$ rRNA A1555 G mutation in three Han Chinese pedigrees. Am J Med Genet A 146A: 1248-1258, 2008.

15. Andrews RM, Kubacka I, Chinerry PF, Lightowlers RN, Turnbull DM and Howell N: Reanalysis and revision of the Cambridge reference sequence for human mitochondrial DNA. Nat Genet 23: 147, 1999.

16. Teng L, Zheng J, Leng J and Ding Y: Clinical and molecular characterization of a Han Chinese family with high penetrance of essential hypertension. Mitochondrial DNA 23: 461-465, 2012.

17. Liu XL, Zhou X, Zhou J, Zhao F, Zhang J, Li C, Ji Y, Zhang Y, Wei QP, Sun YH, et al: Leber's hereditary optic neuropathy is associated with the T12338C mutation in mitochondrial ND5 gene in six Han Chinese families. Ophthalmology 118: 978-985, 2011.

18. Li R, Ishikawa K, Deng JH, Heman-Ackah S, Tamagawa Y, Yang L, Bai Y, Ichimura K and Guan MX: Maternally inherited nonsyndromic hearing loss is associated with the T7511C mutation in the mitochondrial tRNASerUCN gene in a Japanese family. Biochem Biophys Res Commun 328: 32-37, 2005.

19. Li X, Fischel-Ghodsian N, Schwartz F, Yan Q, Friedman RA and Guan MX: Biochemical characterization of the mitochondrial tRNASer (UCN) T7511C mutation associated with nonsyndromic deafness. Nucleic Acids Res 32: 867-877, 2004.

20. Rossmanith W, Tullo A, Potuschak T, Karwan R and Sbisà E: Human mitochondrial tRNA processing. J Biol Chem 270: 12885-12891, 1995.

21. Silvestri G, Servidei S, Rana M, Ricci E, Spinazzola A, Paris E and Tonali P: A novel mitochondrial DNA point mutation in the tRNA (Ile) gene is associated with progressive external ophtalmoplegia. Biochem Biophys Res Commun 220: 623-627, 1996. 